\title{
Analysis of endometrial thickness patterns and pregnancy outcomes considering 12,991 fresh IVF cycles
}

ShuJie Liao ${ }^{1}$, Renjie Wang ${ }^{1}$, Cheng Hu${ }^{2}$, Wulin Pan ${ }^{2}$, Wei Pan ${ }^{3^{*}}$ (D, Dongyang Yu ${ }^{2^{*}}$ and Lei Jin ${ }^{{ }^{*}}$

\begin{abstract}
Background: Different endometrial patterns have an important effect on the relationship between endometrial thickness (EMT) and clinical pregnancy rate. There is a significant difference in age, selection of cycle protocols, and clinical pregnancy rates among four groups with diverse endometrial patterns.

Methods: This retrospective study aimed to assess the association between EMT on human chorionic gonadotropin (HCG) administration day and the clinical outcome of fresh in vitro fertilization (IVF). The 5th, 50th, and 95th percentiles for EMT were determined as 8, 11, and $14 \mathrm{~mm}$, respectively. Patients were sub-divided into four groups based on their EMT in different endometrial patterns (Group 1:<8 mm; Group 2: $\geq 8$ and $\leq 11 \mathrm{~mm}$; Group 3:> 11 and $\leq 14 \mathrm{~mm}$; Group 4:> $14 \mathrm{~mm}$ ). We divided patients into three groups based on their endometrial pattern and evaluated the correlation between EMT and clinical pregnancy rate.
\end{abstract}

Results: We found a positive correlation between pregnancy rates and EMT in all endometrial patterns. Multiple logistic regression analysis proved age, duration of infertility, cycle protocols, number of embryos transferred, progesterone on HCG day, endometrial patterns, and EMT have significant effects on clinical pregnancy rates. Meanwhile, there was a significant difference in age, selection of cycle protocols, and clinical pregnancy rates among four groups with diverse endometrial patterns.

Conclusions: Different endometrial patterns have an important effect on the relationship between EMT and clinical pregnancy rate.

Keywords: In vitro fertilization, Endometrial thickness, Endometrial pattern, Clinical pregnancy

\section{Background}

IVF technology has helped many people who cannot naturally get pregnant solve their infertility. Many clinical factors are used to evaluate the assisted reproductive

\footnotetext{
*Correspondence: mrpanwei2000@163.com; 1713644253@qq.com; 57657713@qq.com

${ }^{1}$ Department of Obstetrics and Gynecology, Tongji Hospital, Tongji Medical College, Huazhong University of Science and Technology,

Wuhan 430030, Hubei, People's Republic of China

${ }^{2}$ School of Economic and Management, Wuhan University, Wuhan 430072, People's Republic of China

${ }^{3}$ School of Applied Economics, Renmin University of China,

Beijing 100872, People's Republic of China
}

technology (ART) outcomes, including EMT and endometrial pattern, which greatly influence pregnancy outcomes $[15,19]$.

Some researchers have examined the relationship between EMT and pregnancy complications [13, 14, 22]. Previous research indicates that EMT and clinical pregnancy rate were positively correlated $[2,8,10,16,18]$. Holden et al. concluded that EMT $<6 \mathrm{~mm}$ had adverse effects on clinical pregnancy and live birth rates following initial IVF cycles [7]. A meta-analysis by Gao et al. explored whether EMT could predict pregnancy outcomes after IVF. They suggested that lower EMT was 
associated with a lower incidence of pregnancy rate, implantation rate, and live birth or ongoing pregnancy rate [5]. Studies suggest that EMT has little effect on pregnancy outcomes. Bu et al. found that a thick endometrium did not have a detrimental effect on IVF outcome [4].

Zhao et al. [21] stated that EMT and endometrial pattern had no predictive value on the outcome of in vitro fertilization-embryo transfer (IVF-ET) [20]. Weiss et al. suggested that differences in EMT between pregnant women and non-pregnant women were too small to guide individual treatments [17]. However, other studies had opposite conclusions. Researchers found that a homogenous, hypoallergenic endometrium had a detrimental effect on clinical pregnancy rate [6]. Kasius et al. [9] found it hard to identify women unlikely to conceive after IVF based on EMT. A significant relationship between EMT and IVF results was not apparent, and their value remains controversial.

Our study combined EMT and endometrial patterns to analyze their impact on clinical pregnancy rates. Moreover, most of the former research samples primarily included Caucasian subjects, while we focus on Asian individuals. We also have an important sample size and believe our research can further understanding of the relationship between EMT and pregnancy rates. Our patients were divided into three groups based on their endometrial pattern to accurately evaluate the correlation between EMT and clinical pregnancy rate.

\section{Methods}

\section{Patient database}

A retrospective cohort of 12,991 in vitro fertilizationintracytoplasmic sperm injection (IVF-ICSI) cycles was enrolled at the Reproductive Medicine Center of Tongji Hospital, PR China from 2014 to 2017. We included fresh IVF-ICSI cycles and embryo transfers within the study period, regardless of the infertility diagnosis, reproductive history, body mass index, or insemination method. Cycles using donor oocytes or suspect endometrial abnormalities were excluded. The cause of infertility was categorized as ovary-related factors, tubal-related factors, endometriosis, male factors, uterine factors, other (including unexplained factors), and multiple factors. The Data Analysis Center of Tongji Hospital performed all data acquisition, management, and analyses.

The patients provided personal information such as age and duration of infertility. Professional medical technicians obtained other factors, including estradiol (E2) and progesterone levels on day 1 of stimulation, folliclestimulating hormone (FSH), and the number of oocytes retrieved.
All transvaginal ultrasound (TVU) assessments were performed in our center by specialist clinicians using the same standardized protocol on the same ultrasound instrument (ALOKA ColorSound ProSound SSD3500SV, Hitachi Aloka Medical, USA). We measured EMT and endometrial patterns on the medial sagittal plane of the uterus on the day of HCG administration and the maximum thickness from one interface to the other at the junction of the endometrial muscle. Other factors measured include body mass index, cycle protocols, the number of oocytes retrieved, the number of embryos transferred, time of gonadotropins used, the number of mature follicles, HMG dose, and clinical pregnancy rate.

Patients were divided into four groups by EMT: Group 1 ( $\leq 8 \mathrm{~mm})$; Group 2 (8-11 $\mathrm{mm})$; Group 3 (11-14 $\mathrm{mm})$; Group $4(\geq 14 \mathrm{~mm})$. Endometrial patterns were classified as type A (a triple line pattern consisting of a central hyperechoic line surrounded by two hypoechoic layers); type B (an intermediate isoechogenic pattern with the same reflectivity as the surrounding myometrium and a poorly defined central echogenic line); and type $\mathrm{C}$ (homogenous, hypoallergenic endometrium (Zhao et al. 2014). Images of the three endometrial patterns are shown in Fig. 1.

Ovarian stimulation protocols, including the long agonist protocol, short agonist protocol, and prolonged agonist protocol, were carried out as previously described [11, 12],Ai et al. [1]. The long agonist protocol (GnRH agonist protocol) was as follows: $0.1 \mathrm{mg} / \mathrm{d}$ Triptorelin (Diphereline, IPSEN, Paris, France) was subcutaneously injected from the midluteal phase of the last menstrual cycle. Nondiameter follicular cysts $>10 \mathrm{~mm}$ were defined as the reduction criterion. The patients were administered recombination follicle-stimulating hormone at 75-150 IU/day (rFSH, Gonal-F, Merck Serono, Switzerland) while Triptorelin was reduced to $0.05 \mathrm{mg} / \mathrm{d}$.

The short antagonist protocol (GnRH antagonist protocol) was as follows: rFSH stimulation was initiated on day two or three of the menstrual cycle until ovulation induction. During the stimulation, the rFSH dosage ranged from 150 to $-225 \mathrm{IU}$, and was adjusted according to the ovarian response as evaluated by TVU. When the leading follicles reached a mean diameter of $14 \mathrm{~mm}$, Cetrotide (Cetrotide, Merck Serono, Germany) was subcutaneously injected at $0.25 \mathrm{mg} / \mathrm{d}$ until ovulation induction.

The prolonged agonist protocol (GnRH agonist ultralong protocol) was as follows: a single dose of $3.75 \mathrm{mg}$ Triptorelin was administrated on the first or second day of the menstrual cycle. $\mathrm{rFSH}$ was initiated daily 28 days later when the endometrial thickness was $<5 \mathrm{~mm}$, the follicular diameter was $<8 \mathrm{~mm}$, E2 was $<50 \mathrm{pg} / \mathrm{ml}$, and $\mathrm{LH}$ was $<3 \mathrm{IU} / \mathrm{L}$, until ovulation induction. The 

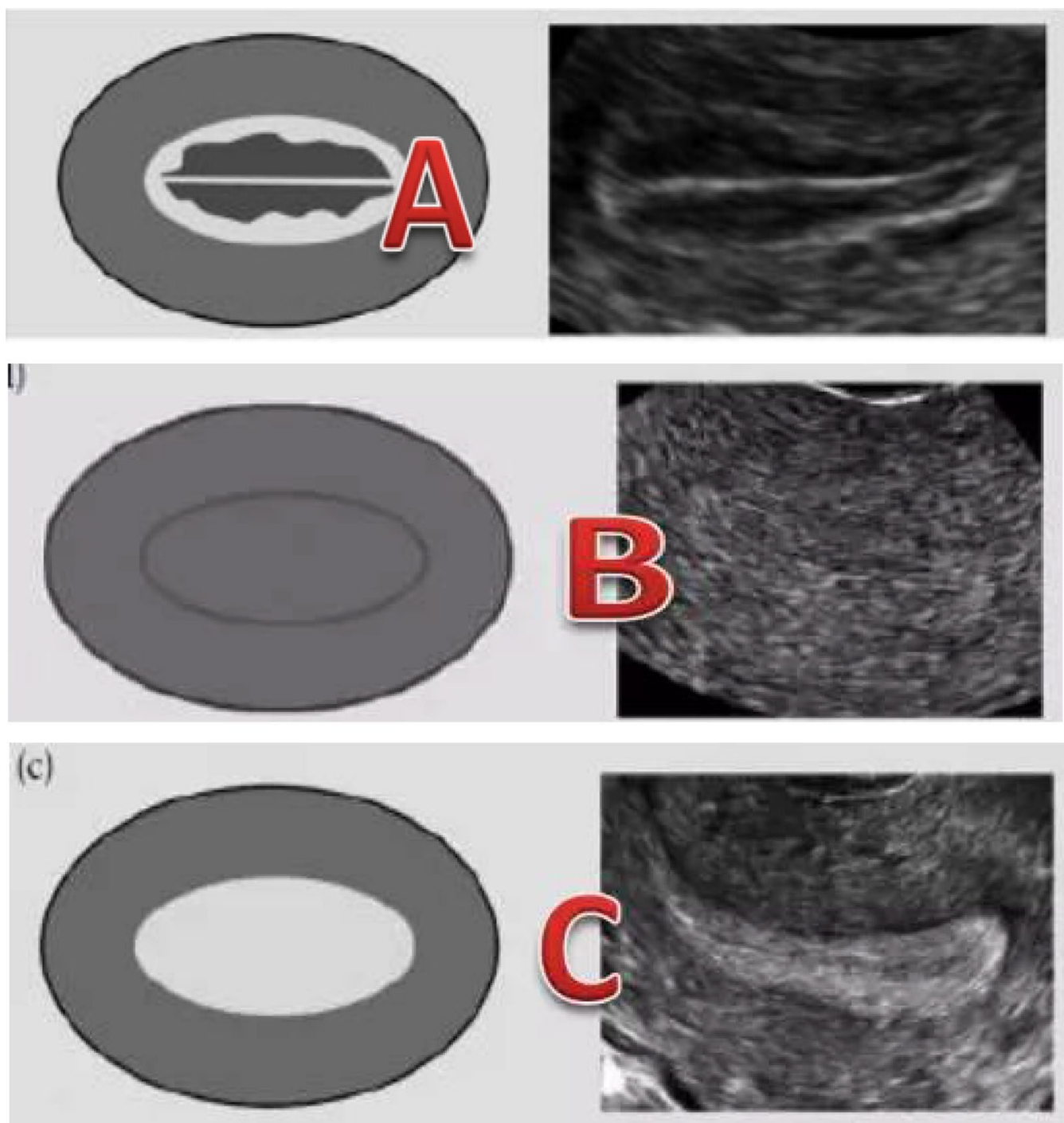

Fig. 1 Schematic diagram of three types of endometrium

gonadotropin dose ranged between 150 and $225 \mathrm{IU}$ and was adjusted based on the ovarian response.

For all three protocols, $0.25 \mathrm{mg}$ of recombinant human chorionic gonadotropin (rhCG) (Ovidrel, Merck Serono, Switzerland) was administered when at least two follicles reached a mean diameter of $18 \mathrm{~mm}$. Oocytes were retrieved $36 \mathrm{~h}$ after rhCG injection by transvaginal ultrasound-guided single-lumen needle aspiration. ICSI was conducted according to sperm quality, considering severe male factor infertility or previous fertilization failures. Luteal phase support was initiated on the first day after oocyte retrieval. Embryo transfer was performed on day two or three after oocyte retrieval. Embryo scoring was performed according to the Istanbul consensus (Alpha
Scientists in Reproductive Medicine and Eshre Special Interest Group Embryology, [3].

\section{Data statement}

As our data belongs to Tongji Hospital, its availability is restricted and we are unauthorized to disclose it. All data acquisition and data management were performed by the Data Analysis Center of Tongji Hospital. The study was approved by the ethics committees at the Reproductive Medicine Center of Tongji Hospital, and informed consent was signed before patient participation. According to the IRB of Tongji Hospital, our study did not require an ethics review because all women participating in the study received routine IVF 
treatment in the hospital and no additional intervention or sampling was performed $[11,12]$.

\section{Main supporting software}

$\mathrm{R}$ language was used for all statistical analyses. Continuous variables were analyzed by Student's $t$-test when categorical variables were checked by the $\chi^{2}$ test. Oneway ANOVA and $\chi^{2}$ tests were used to verify the differences between the four groups. We used a multivariate logistic regression model to assess the prognostic value of the various variables on clinical pregnancy. $P<0.05$ was considered to be significant.

\section{Multivariate logistic regression analysis to determine} the influence of different factors on pregnancy outcomes References and univariate logistic regression usually include age grouping, causes of infertility, EMT, endometrial patterns, FSH, large follicle numbers, treatment plan, number of embryo transfers, number of eggs obtained, and flavonoids as independent variables. Multivariate unconditional regression analysis was performed on whether pregnancy was a dependent variable. Age grouping, causes of infertility, number of years of infertility, basic antral follicle groupings, number of cycles, and pregnancy grouping were selected as the multi-classification covariates (using the definition of variables to analyze the forward method based on partial maximum

Table 1 Multivariate unconditional logistic regression analysis

\begin{tabular}{|c|c|c|c|c|c|c|c|}
\hline Factors & Factors grouping & B & S.E, & Wals & df & Sig & $\operatorname{Exp}(B)$ \\
\hline \multirow[t]{5}{*}{ Age } & $<25$ & & & 83.775 & 4.000 & 0.000 & \\
\hline & $25-30$ & 0.063 & 0.106 & 0.358 & 1.000 & 0.550 & 1.065 \\
\hline & $30-35$ & 0.034 & 0.107 & 0.099 & 1.000 & 0.753 & 1.034 \\
\hline & $35-40$ & -0.183 & 0.118 & 2.386 & 1.000 & 0.122 & 0.833 \\
\hline & $>40$ & -1.375 & 0.186 & 54.716 & 1.000 & 0.000 & 0.253 \\
\hline \multirow[t]{6}{*}{$\mathrm{DOI}$} & Less than 1 year & & & 19.995 & 5.000 & 0.001 & \\
\hline & 1-3 year & -0.081 & 0.132 & 0.380 & 1.000 & 0.538 & 0.922 \\
\hline & 3-6 year & -0.104 & 0.131 & 0.631 & 1.000 & 0.427 & 0.901 \\
\hline & 6-9 year & -0.242 & 0.143 & 2.882 & 1.000 & 0.090 & 0.785 \\
\hline & 9-12 year & -0.422 & 0.167 & 6.363 & 1.000 & 0.012 & 0.656 \\
\hline & 12and above & -0.606 & 0.205 & 8.758 & 1.000 & 0.003 & 0.546 \\
\hline \multirow[t]{4}{*}{ EMT } & Group1 & & & 98.562 & 3.000 & 0.000 & \\
\hline & Group2 & 0.610 & 0.103 & 34.719 & 1.000 & 0.000 & 1.840 \\
\hline & Group3 & 0.806 & 0.104 & 60.242 & 1.000 & 0.000 & 2.239 \\
\hline & Group4 & 1.105 & 0.119 & 86.471 & 1.000 & 0.000 & 3.021 \\
\hline \multirow[t]{3}{*}{ Endometrial pattern } & A & & & 12.339 & 2.000 & 0.002 & \\
\hline & B & -0.229 & 0.068 & 11.199 & 1.000 & 0.001 & 0.795 \\
\hline & $C$ & -0.135 & 0.088 & 2.319 & 1.000 & 0.128 & 0.874 \\
\hline \multirow[t]{4}{*}{ Mature follicle number } & $<5$ & & & 10.745 & 3.000 & 0.013 & \\
\hline & $5-9$ & 0.219 & 0.096 & 5.214 & 1.000 & 0.022 & 1.245 \\
\hline & $10-14$ & 0.326 & 0.102 & 10.236 & 1.000 & 0.001 & 1.385 \\
\hline & $\geq 15$ & 0.271 & 0.117 & 5.365 & 1.000 & 0.021 & 1.312 \\
\hline \multirow[t]{5}{*}{ clinical protocol } & Long & & & 47.341 & 3.000 & 0.000 & \\
\hline & Short & -0.232 & 0.069 & 11.325 & 1.000 & 0.001 & 0.793 \\
\hline & Prolong & 0.411 & 0.079 & 26.875 & 1.000 & 0.000 & 1.508 \\
\hline & Other & -0.101 & 0.102 & 0.989 & 1.000 & 0.320 & 0.904 \\
\hline & $P$ & -0.489 & 0.078 & 39.090 & 1.000 & 0.000 & 0.613 \\
\hline \multirow[t]{5}{*}{ Number of embryo transfer } & 0 (frozen) & & & 194.106 & 3.000 & 0.000 & \\
\hline & 1 & 20.154 & 511.654 & 0.002 & 1.000 & 0.969 & $565,920,737.626$ \\
\hline & 2 & 21.041 & 511.654 & 0.002 & 1.000 & 0.967 & $1,373,929,027.313$ \\
\hline & 3 & 20.499 & 511.654 & 0.002 & 1.000 & 0.968 & $799,202,249.339$ \\
\hline & constant & -21.098 & 511.654 & 0.002 & 1.000 & 0.967 & 0.000 \\
\hline
\end{tabular}


likelihood estimation). The final indicators included in the equation are shown in Table 1.

\section{Prediction model}

The test variable was the predicted successful pregnancy rate obtained by the model. Whether successful pregnancy is the state variable or not, the sensitivity is ordinate and the 1-specificity is the ROC curve of the abscissa (Fig. 2).

The area under the ROC curve is 0.870 , the standard error is 0.003 , and the $95 \%$ confidence interval is \pm 0.006 . SPSS can be used to obtain the sensitivity and specificity pairs corresponding to different diagnostic thresholds. The graph's coordinate points can be drawn. When $P=0.2452$, the Yoden index is 0.640 , which is considered the diagnostic boundary. The discriminating situation is shown in Table 2.

\section{Results}

A total of 12,991 IVF cycles were included in our study and the clinical pregnancy rate was $28.1 \%$ (3656). Demographic data are presented in Table 3. Continuous variables are presented as means and standard deviations and categorical variables are presented as a frequency. The subjects in the pregnancy group were younger, usually chose long agonist protocols, and used lower doses of HMG, E2, and progesterone. Moreover, they had shorter periods of infertility, more embryos transferred, fewer mature follicles, a thicker endometrium, shorter gonadotropin $(\mathrm{Gn})$ time, and were more type-A than

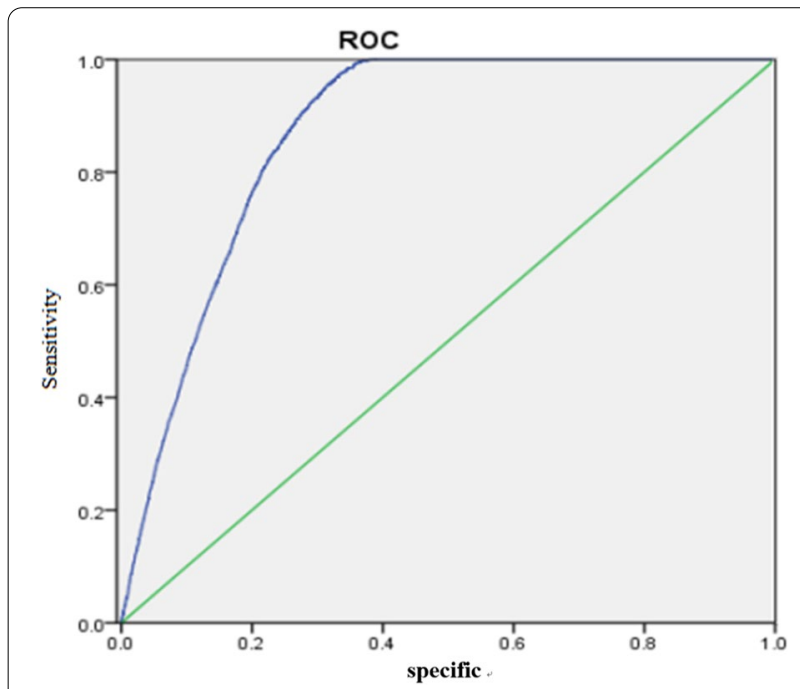

Fig. 2 ROC curve. Note: The predicted value of the successful pregnancy rate is the test variable. Whether the successful pregnancy is the state variable, the sensitivity is the ordinate, and the 1 -specificity is the ROC curve of the abscissa. The area under the ROC curve is equal to 0.870
Table 2 Diagnostic test table

\begin{tabular}{lllr}
\hline Predicting IVF results & pregnant & Not pregnant & Total \\
\hline Successful pregnancy & $3562(\mathrm{TP})$ & $3123(\mathrm{FP})$ & 6685 \\
Not pregnant & $94(\mathrm{FN})$ & $6212(\mathrm{TN})$ & 6306 \\
Total & 3656 & 9335 & 12,991 \\
\hline
\end{tabular}

TP actually successful pregnancy and identify the period of successful pregnancy

FN actually successful pregnancy but discriminates the period of unsuccessful pregnancy

FP actually unsuccessful pregnancy but discriminates the cycle of successful pregnancy

TN actually unsuccessful pregnancy and discriminates the period of unsuccessful pregnancy

$\mathrm{N}=\mathrm{TP}+\mathrm{FN}+\mathrm{FP}+\mathrm{TN}$

Sensitivity: $\mathrm{TP} / \mathrm{TP}+\mathrm{FN}=97.42 \%$

Specificity: TN/FP +TN $=66.54 \%$

Correct rate $=\mathrm{TP}+\mathrm{TN} / \mathrm{N}=75.23 \%$

that subjects in the non-pregnancy group (all variables showed significant statistical differences between the two groups). Both groups had a similar BMI and number of oocytes retrieved (no statistically significant differences). Table 4 shows the primary infertility diagnosis for subjects in both groups.

\section{Statistics of patients with different endometrial patterns}

Table 5 summarizes statistics for patients with triple line patterns $(\mathrm{N}=8555)$, which was significantly different from the logistic regression analyses. Patients in the four different EMT groups had similar progesterone levels $(P>0.0 .5)$. There was a negative association between age and EMT ( $34.42 \pm 6.02$ vs. $31.32 \pm 5.00$ vs. $30.4 \pm 4.53$ vs. $30.09 \pm 4.34 ; P<0.001)$. However, the proportion of patients with thinner EMTs Group $1(<8 \mathrm{~mm})$ who chose long agonist protocols was significantly lower $(28.5 \%$ vs. $54.0 \%$ vs. $59 \%$ vs. $60.1 \% ; P<0.001 ; 8.2 \%$ vs. $10.1 \%$ vs. $16.4 \%$ vs. $20.9 \%$; $P<0.001$ ).

Patients with intermediate isoechogenic patterns $(\mathrm{N}=2434)$ had similar progesterone levels $(P>0.05)$ (Table 6). But the number of embryos transferred was significantly lower in patients with a thinner EMT $(0.53$ vs. 0.87 vs. 1.05 vs. $1.07 ; P<0.001)$. Patients in Group 4 were slightly older than those in Group 3 (30.9 vs. 30.8). Clinical pregnancy rates were significantly higher in patients with thicker EMTs ( $7.5 \%$ vs. $18.6 \%$ vs. $27.8 \%$ vs. 35.2\%; $P<0.001$ ).

For patients with a hypoallergenic pattern $(\mathrm{N}=2002)$ (Table 7$)$, there was a negative association between age and EMT (32.4 vs. 31.9 vs. 30.9 vs. 30.7; $P<0.001)$. Unlike other endometrial patterns, these patients had similar infertility durations $(P>0.05)$. 
Table 3 demographic data of potential predictors in pregnant and non-pregnant women

\begin{tabular}{|c|c|c|c|}
\hline Variables & $\begin{array}{l}\text { Pregnancy }(+) \\
(\mathrm{N}=3656)\end{array}$ & $\begin{array}{l}\text { Non-pregnancy }(-) \\
(\mathrm{N}=9335)\end{array}$ & $P$ value \\
\hline Age (years) & $30.18 \pm 4.13$ & $32.42 \pm 5.75$ & $<0.01$ \\
\hline $\mathrm{BMI}\left(\mathrm{kg} / \mathrm{m}^{2}\right)$ & $21.88 \pm 2.93$ & $21.93 \pm 2.90$ & 0.3278 \\
\hline Cycle protocols & & & $<0.01$ \\
\hline Long agonist protocol & $2302(63.0 \%)$ & $4423(47.4 \%)$ & \\
\hline Short agonist protocol & $575(15.7 \%)$ & $1870(20.0 \%)$ & \\
\hline Prolonged agonist protocol & $565(15.5 \%)$ & $857(9.2 \%)$ & \\
\hline Other & $214(5.9 \%)$ & $2185(23.4 \%)$ & \\
\hline Duration of infertility (years) & $3.67 \pm 2.68$ & $4.25 \pm 3.61$ & $<0.01$ \\
\hline $\mathrm{FSH}(\mathrm{mlU} / \mathrm{ml})$ & $7.38 \pm 2.57$ & $8.05 \pm 3.65$ & $<0.01$ \\
\hline embryo transferred & $1.86 \pm 0.34$ & $0.66 \pm 0.87$ & $<0.01$ \\
\hline HMG & $8.05 \pm 7.54$ & $9.74 \pm 8.92$ & $<0.01$ \\
\hline Gntime & $10 \pm 1.78$ & $9.82 \pm 2.05$ & $<0.01$ \\
\hline mature follicles & $9.97 \pm 3.93$ & $9.47 \pm 5.67$ & $<0.01$ \\
\hline endometrial thickness on HCGday & $11.68 \pm 2.38$ & $10.54 \pm 2.81$ & $<0.01$ \\
\hline E2 & $3362.93 \pm 1756.74$ & $3477.31 \pm 2652.94$ & 0.004228 \\
\hline Progesterone (ng/ml) & $0.9 \pm 0.30$ & $1.23 \pm 1.72$ & $<0.01$ \\
\hline oocytes retrieved (n) & $11.31 \pm 4.59$ & $11.33 \pm 7.94$ & 0.8667 \\
\hline endometrial morphology & & & $<0.01$ \\
\hline a & $2801(76.6 \%)$ & $5754(61.6 \%)$ & \\
\hline $\mathrm{b}$ & $539(14.7 \%)$ & $1895(20.3 \%)$ & \\
\hline c & $316(8.6 \%)$ & $1686(18.1 \%)$ & \\
\hline
\end{tabular}

Data are mean \pm SD or number (percentage)

$B M /$ body mass index $\left(\mathrm{kg} / \mathrm{m}^{2}\right)$, FSH follicle-stimulating hormone

a Two-sample t-test

${ }^{b}$ Pearson $x^{2}$ test

Table 4 Diagnostic categories

\begin{tabular}{lcc}
\hline Variables & $\begin{array}{l}\text { Pregnancy (+) } \\
(\mathbf{N}=3656)\end{array}$ & $\begin{array}{l}\text { Non-pregnancy (-) } \\
(\mathbf{N}=\mathbf{9 3 3 5})\end{array}$ \\
\hline Ovary-related factors & $114(3.1 \%)$ & $481(5.2 \%)$ \\
Tubal related factors & $756(20.7 \%)$ & $1599(17.1 \%)$ \\
Endometriosis & $42(1.1 \%)$ & $88(0.9 \%)$ \\
Male factors & $343(9.4 \%)$ & $705(7.6 \%)$ \\
Uterine factors & $37(1 \%)$ & $81(0.8 \%)$ \\
Other & $1344(36.8 \%)$ & $3408(36.5 \%)$ \\
Multiple causes & $1020(27.9 \%)$ & $2973(31.8 \%)$ \\
\hline
\end{tabular}

Clinical pregnancy rates were still significantly higher in patients with thicker EMT $(2.6 \%$ vs. $14.1 \%$ vs. $23.8 \%$ vs. $30.1 \% ; P<0.001)$.

Tables 8, 9 and 10 show the basic indicators and pregnancy results for different EMT groups. The indicators include endometrial pattern, age, duration of infertility, number of embryos transferred, progesterone levels, and pregnancy outcomes for each clinical protocol (long agonist protocol, short agonist protocol, and prolonged agonist protocol).

\section{Influence of different factors on pregnancy outcomes}

Seven indicators were included in the predictive model: age, duration of infertility, EMT, endometrial pattern, number of large follicles, treatment plan, and number of embryos transferred. The optimal regression equation is:

$\hat{Y}=21.587+0.063^{*}$ (for $25-30$ year old group $)+0.034 *$ (for 30-35 year old group) $-0.183 *$ (for 35-40 year old group $)-1.375^{*}$ (for $\geq 40$ year old group) $-0.081 *$ (for $<1$ year) -0.104 * (for $1-3$ years) $-0.242 *$ (for 3-6 years) $-0.422^{*}$ (for 6-9 years) $-0.606^{*}$ (for $\geq 9$ years) $+0.610^{*}$ (intima thickness is 2$)+0.806^{*}$ (intima thickness is 3 ) $+1.105^{*}$ (intima thickness is $4)-0.229 *$ (endometrial pattern B) $-0.135 *$ (endometrial pattern C) $+0.219 *(5-9)+0.326 *(10-14)+0.271 *$ $(\geq 15) \quad-0.232 *$ (short program $)+0.411^{*}$ (long program $)+20.154 *$ (embryo transfer is 1$)+21.041^{*}$ (embryo transfer is 2$)+20.499 *$ (embryo transfer is 3 ).

The probability of a positive result (successful pregnancy): 
Table 5 Predictors and clinical outcome according to EMT with tripleline patterns

\begin{tabular}{|c|c|c|c|c|c|}
\hline Variables & $\begin{array}{l}\text { Group1 } \\
(\mathrm{N}=785)\end{array}$ & $\begin{array}{l}\text { Group2 } \\
(\mathrm{N}=3476)\end{array}$ & $\begin{array}{l}\text { Group3 } \\
(\mathrm{N}=3345)\end{array}$ & $\begin{array}{l}\text { Group4 } \\
(\mathrm{N}=949)\end{array}$ & $P$ value \\
\hline Age (years) & $34.42 \pm 6.02$ & $31.32 \pm 5.00$ & $30.4 \pm 4.53$ & $30.09 \pm 4.34$ & $<0.01$ \\
\hline Duration of infertility (years) & $4.35 \pm 4.05$ & $3.91 \pm 3.16$ & $3.95 \pm 3.01$ & $3.81 \pm 2.79$ & 0.00159 \\
\hline Clinical protocol & & & & & $<0.01$ \\
\hline Long agonist protocol & $224(28.5 \%)$ & $1877(54.0 \%)$ & 1973 (59\%) & $570(60.1 \%)$ & \\
\hline Short agonist protocol & $140(17.8 \%)$ & $837(24.1 \%)$ & $589(17.6 \%)$ & $121(12.8 \%)$ & \\
\hline Prolonged agonist protocol & $64(8.2 \%)$ & $352(10.1 \%)$ & $548(16.4 \%)$ & $198(20.9 \%)$ & \\
\hline Other & $357(45.5 \%)$ & $410(11.8 \%)$ & $235(7.0 \%)$ & $60(6.3 \%)$ & \\
\hline Embryo transferred & $0.65 \pm 0.88$ & $1.13 \pm 0.92$ & $1.21 \pm 0.90$ & $1.18 \pm 0.91$ & $<0.01$ \\
\hline Progesterone(ng/ml) & $1.08 \pm 2.34$ & $1.05 \pm 0.63$ & $1.03 \pm 0.48$ & $1.01 \pm 0.41$ & 0.261 \\
\hline Clinical pregnancy & $96(12.2 \%)$ & $1071(30.8 \%)$ & $1250(37.4 \%)$ & $384(40.5 \%)$ & $<0.01$ \\
\hline
\end{tabular}

a One-way ANOVA. Values are mean +SD

${ }^{b}$ Pearson $X^{2}$ test. Values are number (percentage)

Table 6 Predictors and clinical outcome according to EMT with intermediate isoechogenic pattern

\begin{tabular}{|c|c|c|c|c|c|}
\hline Variables & $\begin{array}{l}\text { Group1 } \\
(\mathrm{N}=427)\end{array}$ & $\begin{array}{l}\text { Group2 } \\
(\mathrm{N}=864)\end{array}$ & $\begin{array}{l}\text { Group3 } \\
(\mathrm{N}=769)\end{array}$ & $\begin{array}{l}\text { Group4 } \\
(\mathrm{N}=374)\end{array}$ & $P$ value \\
\hline Age (years) & $35.86 \pm 5.79$ & $32.54 \pm 5.47$ & $30.85 \pm 5.13$ & $30.93 \pm 4.66$ & $<0.01$ \\
\hline Duration of infertility (years) & $5.17 \pm 4.70$ & $4.37 \pm 3.50$ & $4.41 \pm 3.58$ & $4.23 \pm 3.35$ & 0.000804 \\
\hline Clinical protocol & & & & & $<0.01$ \\
\hline Long agonist protocol & $123(28.8 \%)$ & $499(57.8 \%)$ & $548(71.3 \%)$ & $271(72.5 \%)$ & \\
\hline Short agonist protocol & $63(14.8 \%)$ & $163(18.9 \%)$ & $105(13.7 \%)$ & $52(13.9 \%)$ & \\
\hline Prolonged agonist protocol & $6(1.4 \%)$ & $12(1.4 \%)$ & $13(1.7 \%)$ & $9(2.4 \%)$ & \\
\hline Other & $235(55.0 \%)$ & $190(22.0 \%)$ & $103(13.4 \%)$ & $42(11.2 \%)$ & \\
\hline Embryo transferred & $0.53 \pm 0.81$ & $0.87 \pm 0.92$ & $1.05 \pm 0.93$ & $1.07 \pm 0.92$ & $<0.01$ \\
\hline Progesterone(ng/ml) & $1.49 \pm 2.87$ & $1.51 \pm 3.05$ & $1.24 \pm 1.29$ & $1.18 \pm 1.60$ & 0.0352 \\
\hline Clinical pregnancy & $32(7.5 \%)$ & 161 (18.6\%) & $214(27.8 \%)$ & $132(35.2 \%)$ & $<0.01$ \\
\hline
\end{tabular}

${ }^{a}$ One-way ANOVA. Values are mean + SD

${ }^{b}$ Pearson $X^{2}$ test. Values are number (percentage)

Table 7 Predictors and clinical outcome according to EMT with hypoallergenic pattern

\begin{tabular}{|c|c|c|c|c|c|}
\hline Variables & $\begin{array}{l}\text { Group1 } \\
(\mathrm{N}=549)\end{array}$ & $\begin{array}{l}\text { Group2 } \\
(\mathrm{N}=631)\end{array}$ & $\begin{array}{l}\text { Group3 } \\
(N=543)\end{array}$ & $\begin{array}{l}\text { Group4 } \\
(\mathrm{N}=279)\end{array}$ & $P$ value \\
\hline Age (years) & $37.77 \pm 5.88$ & $34.03 \pm 6.44$ & $31.6 \pm 5.31$ & $31.3 \pm 5.00$ & $<0.01$ \\
\hline Duration of infertility (years) & $4.16 \pm 4.17$ & $4.16 \pm 3.86$ & $4.14 \pm 3.46$ & $4.21 \pm 3.37$ & 0.994 \\
\hline Clinical protocol & & & & & $<0.01$ \\
\hline Long agonist protocol & $49(9.0 \%)$ & $202(32.0 \%)$ & $249(45.9 \%)$ & $140(50.2 \%)$ & \\
\hline Short agonist protocol & $47(8.6 \%)$ & $125(19.8 \%)$ & $136(25.0 \%)$ & $67(24.0 \%)$ & \\
\hline Prolonged agonist protocol & $30(5.5 \%)$ & $60(9.5 \%)$ & $85(15.7 \%)$ & $45(16.1 \%)$ & \\
\hline Other & $423(77.0 \%)$ & $244(38.7 \%)$ & $73(13.4 \%)$ & $27(9.7 \%)$ & \\
\hline Embryo transferred & $0.16 \pm 0.53$ & $0.55 \pm 0.85$ & $0.84 \pm 0.94$ & $0.94 \pm 0.95$ & $<0.01$ \\
\hline Progesterone (ng/ml) & $1.11 \pm 1.21$ & $1.36 \pm 1.91$ & $1.41 \pm 2.12$ & $1.29 \pm 2.61$ & 0.0418 \\
\hline Clinical pregnancy & $14(2.6 \%)$ & $89(14.1 \%)$ & $129(23.8 \%)$ & $84(30.1 \%)$ & $<0.01$ \\
\hline
\end{tabular}

a One-way ANOVA. Values are mean + SD

${ }^{b}$ Pearson $x^{2}$ test. Value are number (percentage) 
Table 8 Predictors and clinical outcome according to EMT with Long agonist protocol

\begin{tabular}{|c|c|c|c|c|c|}
\hline Variables & $\begin{array}{l}\text { Group1 } \\
(\mathrm{N}=396)\end{array}$ & $\begin{array}{l}\text { Group2 } \\
(\mathrm{N}=\mathbf{2 5 7 8})\end{array}$ & $\begin{array}{l}\text { Group3 } \\
(\mathrm{N}=2770)\end{array}$ & $\begin{array}{l}\text { Group4 } \\
(\mathrm{N}=981)\end{array}$ & $P$ value \\
\hline Age (years) & $30.89 \pm 5.88$ & $30.22 \pm 5.02$ & $29.89 \pm 4.44$ & $29.89 \pm 4.56$ & $<0.01$ \\
\hline Duration of infertility (years) & $3.49 \pm 3.98$ & $3.91 \pm 3.16$ & $3.84 \pm 2.95$ & $4.01 \pm 2.55$ & 0.00188 \\
\hline Endometrial patterns & & & & & $<0.01$ \\
\hline A & $224(56.6 \%)$ & $1877(72.8 \%)$ & $1973(71.2 \%)$ & $570(58.1 \%)$ & \\
\hline B & $123(31 \%)$ & 409 (15.9\%) & $548(19.9 \%)$ & $271(27.6 \%)$ & \\
\hline C & 49 (12.4\%) & $202(7.8 \%)$ & $249(9 \%)$ & $140(14.3 \%)$ & \\
\hline Embryo transferred & $1.06 \pm 0.85$ & $1.19 \pm 0.88$ & $1.21 \pm 0.91$ & $1.17 \pm 0.88$ & $<0.01$ \\
\hline Progesterone(ng/ml) & $1.09 \pm 2.22$ & $1.1 \pm 0.44$ & $1.05 \pm 0.42$ & $1.03 \pm 0.38$ & 0.181 \\
\hline Clinical pregnancy & $74(18.7 \%)$ & $831(32.2 \%)$ & $1006(36.3 \%)$ & 391 (39.9\%) & $<0.01$ \\
\hline
\end{tabular}

Table 9 Predictors and clinical outcome according to EMT with short agonist protocol

\begin{tabular}{|c|c|c|c|c|c|}
\hline Variables & $\begin{array}{l}\text { Group1 } \\
(\mathrm{N}=396)\end{array}$ & $\begin{array}{l}\text { Group2 } \\
(\mathrm{N}=2578)\end{array}$ & $\begin{array}{l}\text { Group3 } \\
(\mathrm{N}=\mathbf{2 7 7 0})\end{array}$ & $\begin{array}{l}\text { Group4 } \\
(\mathrm{N}=981)\end{array}$ & $P$ value \\
\hline Age (years) & $30.89 \pm 5.88$ & $30.22 \pm 5.02$ & $29.89 \pm 4.44$ & $29.89 \pm 4.56$ & $<0.01$ \\
\hline Duration of infertility (years) & $3.49 \pm 3.98$ & $3.91 \pm 3.16$ & $3.84 \pm 2.95$ & $4.01 \pm 2.55$ & 0.00188 \\
\hline Endometrial patterns & & & & & $<0.01$ \\
\hline A & $224(56.6 \%)$ & $1877(72.8 \%)$ & $1973(71.2 \%)$ & $570(58.1 \%)$ & \\
\hline B & $123(31 \%)$ & 409 (15.9\%) & 548 (19.9\%) & $271(27.6 \%)$ & \\
\hline C & $49(12.4 \%)$ & $202(7.8 \%)$ & $249(9 \%)$ & $140(14.3 \%)$ & \\
\hline Embryo transferred & $1.06 \pm 0.85$ & $1.19 \pm 0.88$ & $1.21 \pm 0.91$ & $1.17 \pm 0.88$ & $<0.01$ \\
\hline Progesterone(ng/ml) & $1.09 \pm 2.22$ & $1.1 \pm 0.44$ & $1.05 \pm 0.42$ & $1.03 \pm 0.38$ & 0.181 \\
\hline Clinical pregnancy & $74(18.7 \%)$ & $831(32.2 \%)$ & $1006(36.3 \%)$ & 391 (39.9\%) & $<0.01$ \\
\hline
\end{tabular}

Table 10 Predictors and clinical outcome according to EMT with prolonged agonist protocol

\begin{tabular}{|c|c|c|c|c|c|}
\hline Variables & $\begin{array}{l}\text { Group1 } \\
(\mathrm{N}=100)\end{array}$ & $\begin{array}{l}\text { Group2 } \\
(\mathrm{N}=424)\end{array}$ & $\begin{array}{l}\text { Group3 } \\
(\mathrm{N}=646)\end{array}$ & $\begin{array}{l}\text { Group4 } \\
(\mathrm{N}=252)\end{array}$ & $P$ value \\
\hline Age (years) & $31.81 \pm 5.93$ & $30.62 \pm 4.83$ & $29.8 \pm 4.34$ & $29.76 \pm 4.39$ & $<0.01$ \\
\hline Duration of infertility (years) & $3.49 \pm 3.71$ & $3.51 \pm 3.24$ & $3.72 \pm 2.77$ & $3.42 \pm 2.55$ & 0.00103 \\
\hline Endometrial patterns & & & & & $<0.01$ \\
\hline A & $64(64 \%)$ & $352(83 \%)$ & $548(84.8 \%)$ & 198 (78.62\%) & \\
\hline B & $6(6 \%)$ & $12(2.8 \%)$ & $13(2 \%)$ & $9(3.6 \%)$ & \\
\hline C & $30(30 \%)$ & $60(14.2 \%)$ & $85(13.2 \%)$ & $45(17.9 \%)$ & \\
\hline Embryo transferred & $0.91 \pm 0.72$ & $1.13 \pm 0.83$ & $1.16 \pm 0.78$ & $1.17 \pm 0.91$ & $<0.01$ \\
\hline Progesterone(ng/ml) & $1.23 \pm 1.99$ & $1.14 \pm 0.44$ & $1.11 \pm 0.42$ & $1.07 \pm 0.36$ & 0.196 \\
\hline Clinical pregnancy & $25(25 \%)$ & $166(39.2 \%)$ & $270(41.8 \%)$ & $104(41.2 \%)$ & $<0.01$ \\
\hline
\end{tabular}

$$
P=\frac{1}{1+\exp (-\hat{Y})}
$$

The likelihood ratio test statistic of this model is 9395.554, the degree of freedom is $24, P<0.001$, and the regression equation is highly significant. The
Hosmer-Lemeshow goodness-of-fit test statistic of this model is 6.455 , and the $P$ value is equal to 0.596 , which indicates that there is no statistically significant difference between the expected frequency and the observed frequency obtained by the prediction probability. In other words, the model fits well. 
We compared the effects of three different endometrial patterns on pregnancy outcomes (Fig. 3). Significant differences were reported in the clinical pregnancy rate, as follows:

- Pattern A: group 1 (12.2\%), group 2 (30.8\%), group 3 (37.4\%), and group 4 (40.5\%);

- Pattern B: group 1 (7.5\%), group 2 (18.6\%), group 3 (27.8\%), and group 4 (35.2\%); and

- Pattern C: group 1 (2.6\%), group 2 (14.1\%), group 3 (23.8\%), and group 4 (30.1\%).

Multivariate logistic regression analysis (Table 11) indicates that cycle protocols (short agonist protocol: OR 0.7; 95\% CI 0.62-0.8), EMT (OR 1.12; 95\% CI 1.09-1.14), age (OR 0.96; 95\% CI 0.94-0.97), duration of infertility (OR 0.97; 95\% CI 0.95-0.98), progesterone levels (OR 0.63; 95\% CI 0.54-0.73), number of embryos transferred (OR 7.12; 95\% CI 6.52-7.77), and endometrial pattern (pattern B: OR 0.8; 95\% CI 0.7-0.92) were predictive of pregnancy outcomes.

Previous research has mentioned a relationship between abortion and EMT, so we analyzed this too (Fig. 4). When the EMT was less than $6 \mathrm{~mm}$, the miscarriage rate was as high as $50 \%$, and tended to be stable as the EMT increased $(P<0.05)$.

\section{Prediction results}

We calculated the probability of pregnancy in each treatment cycle before the patient entered treatment. We sorted each predicted value from smallest to largest, then used each decile as a cutoff to sort the
Table 11 Logistic Regression Analysis for the association between potential predictors and clinical pregnancy

\begin{tabular}{llc}
\hline Predictors & OR $(95 \% \mathrm{Cl})$ & $P$ \\
\hline Clinical protocol & $*$ & $<0.001 \mathrm{a}$ \\
Long agonist protocol & $0.7(0.62,0.8)$ & $<0.001$ \\
Short agonist protocol & $1.4(1.2,1.64)$ & $<0.001$ \\
Prolonged agonist protocol & $0.85(0.7,1.03)$ & 0.1 \\
Other & $0.97(0.95,0.98)$ & $<0.001$ \\
Duration of infertility & $1.12(1.09,1.14)$ & $<0.001 \mathrm{a}$ \\
Endometrial thickness & & $<0.001$ \\
Endometrial morphology & $*$ & \\
a & $0.8(0.7,0.92)$ & 0.001 \\
b & $0.72(0.61,0.86)$ & $<0.001$ \\
C & $0.96(0.94,0.97)$ & $<0.001$ \\
Age & $0.63(0.54,0.73)$ & $<0.001$ \\
Progesterone & $7.12(6.52,7.77)$ & $<0.001$ \\
ET & &
\end{tabular}

${ }^{*}$ Reference group

a $\mathrm{P}$-value of each variables overall effects after adjusting for the other variables

${ }^{b} \mathrm{P}$-value between each variable subgroups and reference group

"ET" means number of embryos transferred

predicted probabilities into groups. We used the nine decile points to obtain $0.0000,0.0000,0.0000,0.0000$, $0.2845,0.4218,0.5227,0.5742$, and 0.6255 and divided the predicted probability values into 10 groups. As the sample size was too large, we found the first four $P$ values were close to zero after we sorted them from smallest to largest. We combined them into one group for a total of six groups.

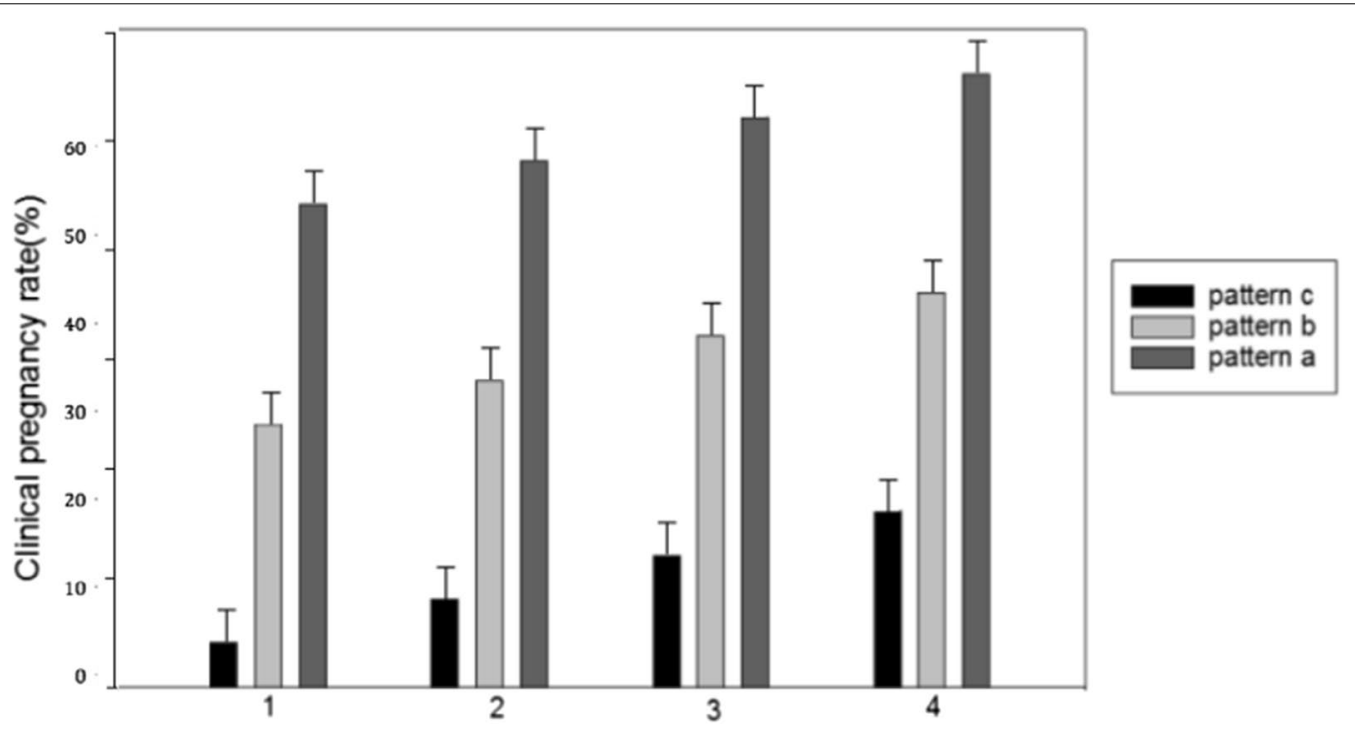

Fig. 3 Distribution of pregnancy rates of three endometrium types in four groups of patients 


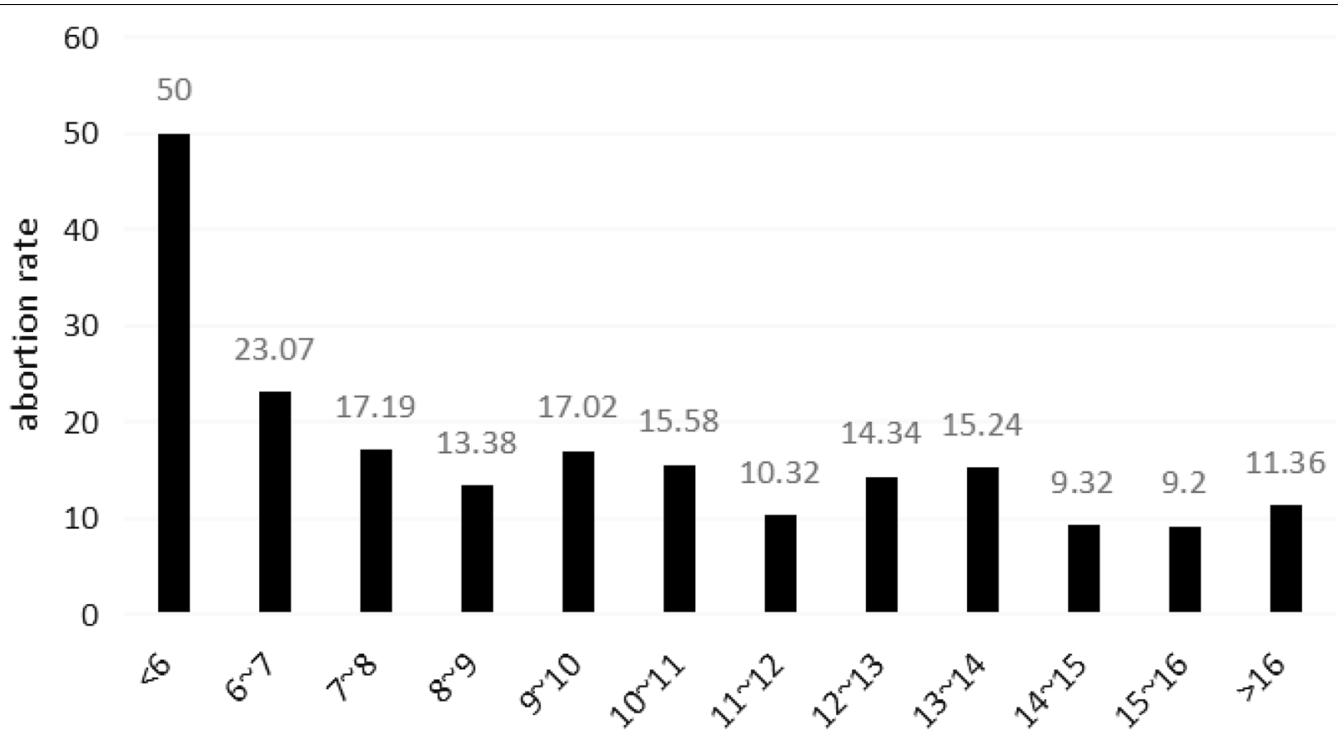

Fig. 4 Predictions of miscarriage rate at different EMT thresholds

EMT

The actual pregnancy rate can be obtained by calculating the number of actual pregnancies in the group and the ratio of the total number of cases in each group. The average predicted pregnancy rate and the average predicted number of pregnancies were calculated in each group. The actual number of pregnancies per group (average predicted number of pregnancies) can be used to obtain the error rate for each group of predictions (Table 12). The prediction error rate of each group was within $\pm 4 \%$, and the maximum error was $3.02 \%$ for the third group. The overall prediction error rate is 0.41 . The average prediction error of each group is taken as an absolute value, $0.72 \%$. In the coordinate system, the average predicted pregnancy rate is used as the axis coordinate point, and the actual observed pregnancy rate value is plotted as the axis coordinate point. The correlation equation is obtained by bivariate correlation using the average predicted pregnancy rate and the actual pregnancy rate of each group (Fig. 5). The correlation coefficient was calculated to obtain $\mathrm{R}^{2}=1, P<0.001$, indicating that there is a close correlation between the predicted pregnancy rate averages.

\section{Discussion}

\section{Main findings}

In this retrospective study, we listed many possible predictors of pregnancy outcome for 12,991 patients undergoing IVF-ISCI. Our study demonstrated that EMT and clinical pregnancy rates are positively correlated. The logistic regression analysis showed that, within all of the other relative IVF factors, EMT was an independent and vital predictor of pregnancy outcomes.

Table 12 Predicted pregnancy rate actual pregnancy rate between groups

\begin{tabular}{|c|c|c|c|c|c|c|c|}
\hline Group & $\begin{array}{l}\text { Predicted } \\
\text { pregnancy rate } \\
(\%)\end{array}$ & $\begin{array}{l}\text { Average predicted } \\
\text { pregnancy rate (\%) }\end{array}$ & $\begin{array}{l}\text { Average predicted } \\
\text { number of infants }\end{array}$ & $\begin{array}{l}\text { Actual } \\
\text { pregnancy }\end{array}$ & $\begin{array}{l}\text { The total number of } \\
\text { cycles in the group }\end{array}$ & $\begin{array}{l}\text { Actual } \\
\text { pregnancy } \\
\text { rate (\%) }\end{array}$ & Error rate (\%) \\
\hline 1 & $<28.45$ & 2.26 & 147 & 151 & 6499 & 2.32 & -0.06 \\
\hline 2 & $28.45-42.18$ & 35.81 & 466 & 466 & 1301 & 35.81 & 0 \\
\hline 3 & $42.18-52.27$ & 48.07 & 614 & 653 & 1278 & 51.09 & -3.02 \\
\hline 4 & $52.27-57.42$ & 55.06 & 730 & 728 & 1325 & 54.94 & 0.01 \\
\hline 5 & $57.42-62.55$ & 59.67 & 766 & 781 & 1284 & 60.82 & -1.15 \\
\hline 6 & $>62.55$ & 67.35 & 879 & 877 & 1304 & 67.25 & 0.10 \\
\hline Total & $<62.55$ & 27.73 & 3602 & 3656 & 12,991 & 28.14 & -0.41 \\
\hline
\end{tabular}




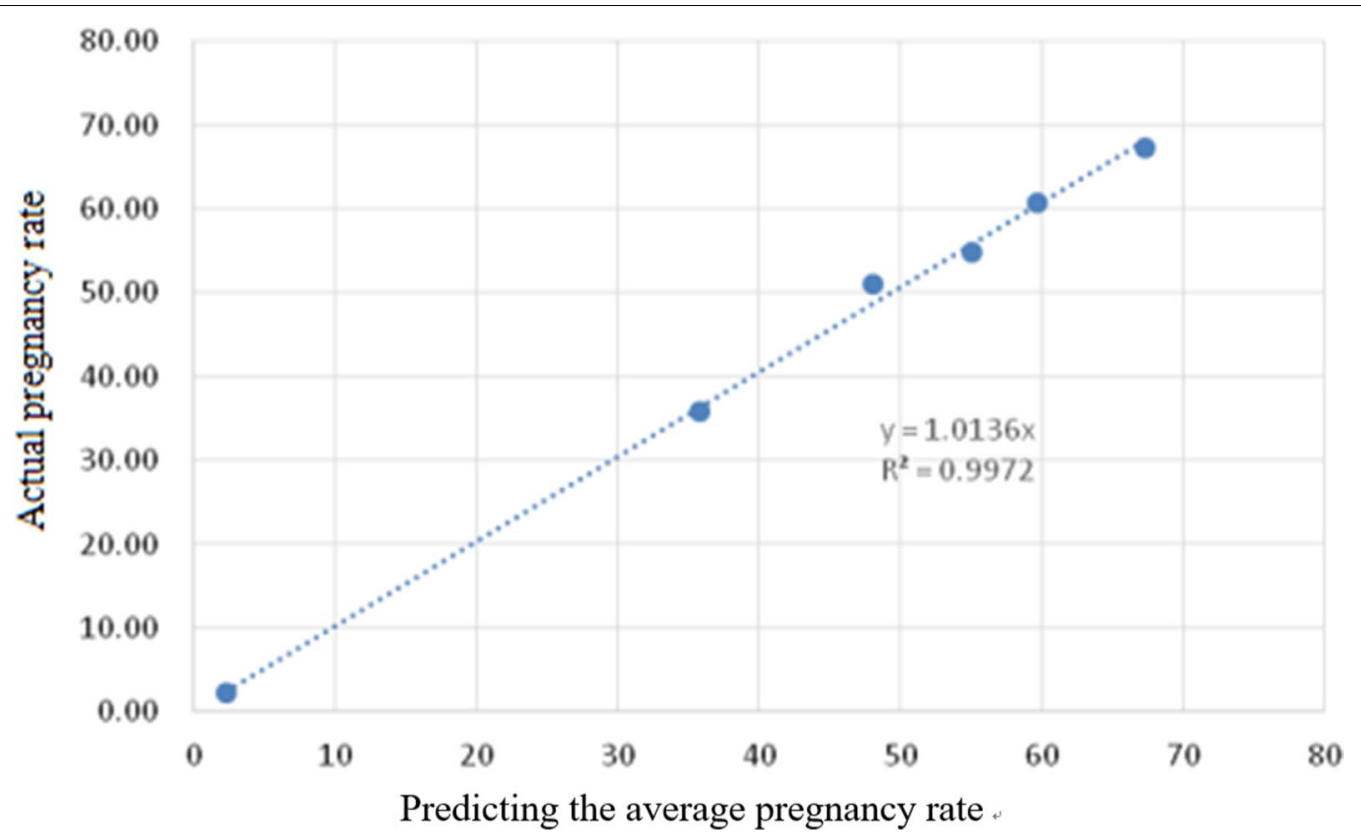

Fig. 5 Estimation of the average pregnancy rate and the actual observed pregnancy rate scatter plot. The correlation equation shows the statistic relationship between the average value of the predicted pregnancy rate and the actual pregnancy rate of each group, and the correlation coefficient is calculated to obtain $R^{2}=1, P<0.001$, indicating that there is a correlation between the predicted pregnancy rate averages and the correlation

In our study, we used $8 \mathrm{~mm}$ as the cut-off value for a thin endometrium. The pregnancy rate increased rapidly when EMT reached $8 \mathrm{~mm}$ (pattern A: $12.2 \%$ vs. $30.8 \%$; pattern B: $7.5 \%$ vs. $18.6 \%$; pattern C: $2.6 \%$ vs. $14.1 \%)$. Patients with thinner EMT were significantly older. Pattern $\mathrm{C}$ on the trigger day suggested a significantly low clinical pregnancy rate. We found a consistent positive correlation between EMT and clinical pregnancy rate in patients with pattern $B$ or pattern C. When the EMT was below $14 \mathrm{~mm}$, the pregnancy rate ranking among the different endometrial patterns was pattern $\mathrm{A}>$ pattern $\mathrm{B}>$ pattern $\mathrm{C}$. When EMT reached $14 \mathrm{~mm}$, clinical pregnancy rates were similar in the three different endometrial patterns.

For patients with different endometrial patterns, the factors of age, duration of infertility, clinical protocol choice, progesterone dose, and the number of embryos transferred were compared between the four groups. Only the clinical protocol choice indicated significant differences in every type of endometrium. Patients with prolonged agonist protocol had a rather low clinical pregnancy rate. The clinical pregnancy rate did not improve with the increase in EMT. However, most patients who chose the long agonist protocol had better clinical pregnancy rates. Therefore, despite other possible factors, the long agonist protocol was recommended in most situations.

\section{Strengths and limitations}

Our retrospective study is more comprehensive than previous research. We included patients with poor physical condition (a BMI range from 14.7 to 43, age range from 20 to 51) for more convincing results. Moreover, few studies have compared pregnancy rate and EMT in three different endometrial patterns. However, the relationship between a thick endometrium and clinical pregnancy rate is controversial. We found that the two vital predictors should be discussed separately. Most previous studies only considered treatment with a standard GnRH-a long protocol. In our study, we examined the long agonist protocol, short agonist protocol, and prolonged agonist protocol.

Our study had some limitations. All indicators, such as E2, progesterone, EMT, and endometrial patterns, were measured on the same day. The predictive values at other time points (baseline, day three of stimulation, etc.) should be examined. 


\section{Conclusion}

We established and evaluated a predictive model of the factors influencing pregnancy rate. The predictive model has excellent discriminative ability regarding the patient's final pregnancy outcome. The pregnancy probability of each cycle calculated by the predictive model roughly reflects the true pregnancy probability of the patient. The smaller the prediction error, the more accurate the prediction result. Therefore, the established model can be used to assess patient prognosis before certain treatments. Patients can be classified as having a low to high pregnancy rate. This model provides a means of communication and decision-making for doctors and patients.

Our examination of the relationship between EMT and IVF outcomes revealed that different endometrial patterns exhibited different characteristics. Factors such as age, cycle protocols, duration of infertility, progesterone levels, and the number of embryos transferred played an important role in predicting pregnancy outcomes. When the EMT is less than $6 \mathrm{~mm}$, we should remind the patient that their miscarriage rate is high. Thick EMT positively affected clinical pregnancy rates, which did not drop when EMT reached $14 \mathrm{~mm}$. This was consistent with some studies that demonstrated no reduction in pregnancy rate in cases of a very thick endometrium [18].

\section{Abbreviations \\ EMT: endometrial thickness; IVF: in vitro fertilization; HCG: human chorionic gonadotropin; ART: assisted reproductive technology; TVU: all transvaginal ultrasound; Gn: gonadotropin.}

\section{Acknowledgements}

The authors would like to thank all the members of the Reproductive Medicine Center of Tongji Hospital that provide us with data and technical support.

\section{Authors' contributions}

W.P., D.Y.Y., L.J., and S.J.L. took part in study design, R.J.W., W. P., S.J.L. collected and analyzed data, C.H., S.J.L. interpreted results, W.P., D.Y.Y., L.J. and S.J.L. wrote the manuscript. All authors read and approved the final manuscript.

\section{Funding}

This study is supported by the National Natural Science Foundation of China (NSFC) (Grant Nos. 81672085, 71871169, U1933120, 81372804). the Chinese medical association of clinical medicine special funds for scientific research projects (17020400709) and the Hubei Provincial Natural Science Foundation of China (2019CFA062); The funders had no role in study design, data collection and analysis, decision to publish, or preparation of the manuscript.

\section{Availability of data and material}

The data generated or analyzed during this study is available from the corresponding author upon reasonable request.

\section{Declarations}

\section{Ethics approval and consent to participate}

The study was approved by the ethics committees at the Reproductive Medicine Center of Tongji Hospital, and informed consent was signed by the patients before participation.

\section{Consent for publication}

Not Applicable.

\section{Competing interests}

The authors have declared that there is no competing interest.

Received: 18 July 2020 Accepted: 23 May 2021

Published online: 03 June 2021

\section{References}

1. Ai J, Jin L, Zheng Y, Gong J, Chen B, Dong X. The estradiol/oocyte ratio predicts the MII oocyte rate, but not live-birth rate in a depot gonadotropin-releasing hormone agonist long protocol. Int J Gynaecol Obst Off Organ Int Fed Gynaecol Obst. 2020. https://doi.org/10.1002/ijgo.13417.

2. Al-Ghamdi A, Coskun S, Al-Hassan S, Al-Rejjal R, Awartani K. The correlation between endometrial thickness and outcome of in vitro fertilization and embryo transfer (IVF-ET) outcome. Reprod Biol Endocr. 2008;6:37.

3. Alpha Scientists in Reproductive Medicine and Eshre Special Interest Group Embryology. The Istanbul consensus workshop on embryo assessment: proceedings of an expert meeting. Hum Reprod. 2011;22(6):632-46.

4. Bu Z, Sun Y. The impact of endometrial thickness on the day of human chorionic gonadotrophin ( $\mathrm{hCG}$ ) administration on ongoing pregnancy rate in patients with different ovarian response. PLoS ONE. 2016;10:e145703.

5. Gao G, Cui X, Li S, Ding P, Zhang S, Zhang Y. Endometrial thickness with in-vitro fertilization: a meta-analysis. Reprod BioMed Online. 2019. https:// doi.org/10.1016/j.rbmo.2019.09.005.

6. Gingold JA, Lee JA, Rodriguez-Purata J, Whitehouse MC, Sandler B, Grunfeld L, Mukherjee T, Copperman AB. Endometrial pattern, but not endometrial thickness, affects implantation rates in euploid embryo transfers. Fertil Steril. 2015;104:620-8.

7. Holden EC, Dodge LE, Moragianni VA. Endometrial thickness and pregnancy outcomes in IVF cycles: a retrospective review of 6369 cycles. Fertil Steril. 2013;100:S126-7.

8. Karmon AE, Cardozo ER, Petrozza JC. Clomid compared with folliclestimulating hormone treatment: similar follicle number means different success rates. Obstet Gynecol. 2015;125(Suppl 1):60S.

9. Kasius A, Smit JG, Torrance HL, Eijkemans MJC, Mol BW, Opmeer BC, Broekmans FJM. Endometrial thickness and pregnancy rates after IVF: a systematic review and meta-analysis. Hum Reprod Update. 2014;20:530-41.

10. Kovacs P, Matyas S, Boda K, Kaali SG. The effect of endometrial thickness on IVF/ICSI outcome. Hum Reprod. 2003;18:2337-41.

11. Pan W, Tu H, Jin L, Hu C, Xiong J, Pan W, Yu D, Wang R, Li Y, Huang W, Liao S. Comparison of recombinant and urinary follicle-stimulating hormones over 2000 gonadotropin-releasing hormone antagonist cycles: a retrospective study. Sci Rep UK. 2019;9:5329.

12. Pan W, Tu H, Jin L, Hu C, Li Y, Wang R, Huang W, Liao S. Decision analysis about the cost-effectiveness of different in vitro fertilization-embryo transfer protocol under considering governments, hospitals, and patient. Medicine (Baltimore). 2019;98(19):e15492. https://doi.org/10.1097/MD. 0000000000015492

13. Rombauts L, McMaster R, Motteram C, Fernando S. Risk of ectopic pregnancy is linked to endometrial thickness in a retrospective cohort study of 8120 assisted reproduction technology cycles. Hum Reprod. 2015;30:2846-52.

14. Rombauts L, Motteram C, Berkowitz E, Fernando S. Risk of placenta praevia is linked to endometrial thickness in a retrospective cohort study of 4537 singleton assisted reproduction technology birthst. Hum Reprod. 2014;29:2787-93.

15. Sharman MJ, Tolcher MC, Laughlin-Tommaso S. Accuracy of endometrial thickness for predicting endometrial polyps [13M]. Obstet Gynecol. 2016:127:109S

16. Singh N, Yadav A, Perumal V. Role of three dimensional endometrial volume and thickness in predicting the outcome of IVF cycles. Fertil Steril. 2016;106:e224.

17. Weiss NS, van Vliet MN, Limpens J, Hompes PGA, Lambalk CB, Mochtar $\mathrm{MH}$, van der Veen F, Mol BWJ, van Wely M. Endometrial thickness in 
women undergoing IUI with ovarian stimulation. How thick is too thin? A systematic review and meta-analysis. ObstetrGynecol Surv. 2017;72(8):484-485. https://doi.org/10.1097/01.ogx.0000521775.45808. 27.

18. Wu Y, Gao X, Lu X, Xi J, Jiang S, Sun Y, Xi X. Endometrial thickness affects the outcome of in vitro fertilization and embryo transfer in normal responders after $\mathrm{GnRH}$ antagonist administration. Reprod Biol Endocrin. 2014;12:96.

19. Yuan X, Saravelos SH, Wang Q, Xu Y, Li T, Zhou C. Endometrial thickness as a predictor of pregnancy outcomes in 10787 fresh IVF-ICSI cycles. Reprod Biomed Online. 2016;33:197-205.

20. Zhao J, Zhang Q, Li Y. The effect of endometrial thickness and pattern measured by ultrasonography on pregnancy outcomes during IVF-ET cycles. Reprod Biol Endocrin. 2012;10:100.
21. Zhao J, Zhang Q, Wang Y, Li Y. Endometrial pattern, thickness and growth in predicting pregnancy outcome following 3319 IVF cycle. Reprod BioMed Online. 2014;29(2):291-98.

22. Zheng Y, Li Z, Xiong M, Luo T, Dong X, Huang B, Zhang H, Ai J. Hormonal replacement treatment improves clinical pregnancy in frozen-thawed embryos transfer cycles: a retrospective cohort study. Am J Transl Res. 2013;6:85-90

\section{Publisher's Note}

Springer Nature remains neutral with regard to jurisdictional claims in published maps and institutional affiliations.
Ready to submit your research? Choose BMC and benefit from:

- fast, convenient online submission

- thorough peer review by experienced researchers in your field

- rapid publication on acceptance

- support for research data, including large and complex data types

- gold Open Access which fosters wider collaboration and increased citations

- maximum visibility for your research: over $100 \mathrm{M}$ website views per year

At BMC, research is always in progress.

Learn more biomedcentral.com/submissions 